\title{
Photosynthesis
}

\section{Electron-transfer theory in question}

Jim Barber

THE reactions by which light is captured and stored as chemical energy in photosynthesis are distinguished by the extremely high quantum yield of the primary conversion process. On page 190 of this issue'. Fleming et al. present data which provide new insights into this process. Using ultrafast spectroscopy, these authors have been able to detect the rate of formation of the initial charged transfer state in the isolated reaction centres of purple photosynthetic bacteria. This occurs in just a few picoseconds and, surprisingly, increases as the temperature is lowered to that of liquid helium.

Their results emphasize that a complete theoretical description of this remarkable primary reaction will require a better understanding of how the protein environment around the reactants influences the very fast electron-transfer rate. One particular challenge which emerges from their observations is to explain, in terms of quantum mechanics, how an intermediate bacteriochlorophyll molecule aids the primary charge separation.

\section{Reaction centre}

All photosynthetic organisms have similar molecular machinery for catalysing the primary storage processes of photosynthesis. The machinery is contained within a protein complex embedded in a membrane and called a reaction centre. The reaction centre is excited by the energy of a photon which is initially absorbed by an antenna system of many hundreds of pigment molecules. The energy of the absorbed photon is rapidly transferred by exciton migration to the reaction centre which acts as a trap. All types of reaction centres contain either chlorophyll (oxygenic organisms, such as higher plants and algae) or bacteriochlorophyll (anoxygenic organisms, such as photosynthetic bacteria) which acts as a primary electron donor, $\mathrm{P}$. When excited, $P$ rapidly gives an electron to a primary electron acceptor, $\mathrm{A}_{1}$, and a charged transfer state or radical pair, $\mathbf{P}^{+} \mathbf{A}_{1}^{-}$, is created.

$$
\mathrm{PA}_{1} \stackrel{h v}{\rightarrow} \mathrm{P}^{*} \mathrm{~A}_{1} \rightarrow \mathrm{P}^{+} \mathrm{A}_{1}^{-}
$$

The transfer of an electron from $\mathrm{P}^{\circ}$ to $\mathrm{A}_{1}$ must be very fast to avoid de-excitation by other routes such as by fluorescence. Also, for the photosynthetic reaction centre to have a very high quantum yield of energy conversion, there must be a negligible back-reaction between $\mathrm{P}^{+}$and $\mathrm{A}_{1}^{-}$. The latter is achieved by rapidly transferring the electron away from $A_{1}^{-}$, to secondary electron acceptors $A_{2}, A_{3}$ and so on, and by passing an electron to $\mathrm{P}^{+}$from the secondary donors $\mathrm{D}_{1}$ and so on.

$$
\begin{gathered}
\mathrm{D}_{1} \mathrm{P}^{+} \mathrm{A}_{1}^{-} \mathrm{A}_{2} \mathrm{~A}_{3} \rightarrow \mathrm{D}_{1} \mathrm{P}^{+} \mathrm{A}_{1} \mathrm{~A}_{2}^{-} \mathrm{A}_{3} \rightarrow \\
\mathrm{D}_{1} \mathrm{P}^{+} \mathrm{A}_{1} \mathrm{~A}_{2} \mathrm{~A}_{3}^{-} \rightarrow \mathrm{D}_{1}^{+} \mathrm{PA}_{1} \mathrm{~A}_{2} \mathrm{~A}_{3}^{-}
\end{gathered}
$$

For some years now, the best experimental system to study these primary electron-transfer processes has been reaction centres isolated from purple photosynthetic bacteria. The importance

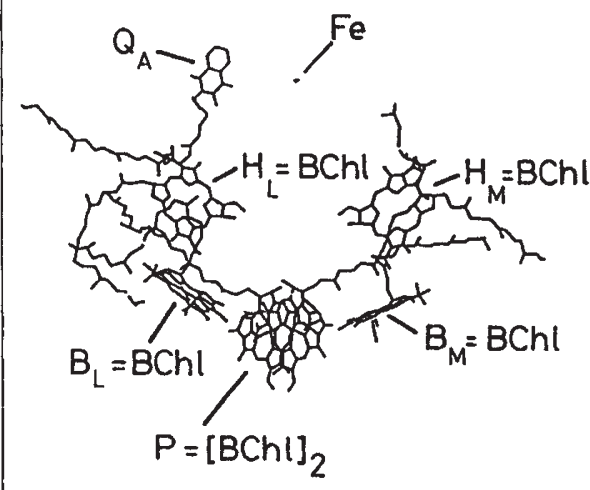

Organization of the prosthetic groups within the reaction centre of Rps. viridis (from ref. 2). The 2-fold symmetry axis is aligned vertically in the plane of the paper. Note that, unlike the reaction centre structure of $R b$. sphaeroides ${ }^{3}$, the secondary quinone $Q_{B}$ is not present but its position is symmetrically related, around the 2-fold axis, to the $Q_{A}$ site.

of this experimental system has been particularly elevated by the recent determination of the three-dimensional structure, to a resolution of atomic distance, of the reaction centres of $R$ hodopseudomonas viridis ${ }^{2}$ and Rhodobacter sphaeroides $^{3}$. These outstanding studies indicate that there are two different symmetrically related possible pathways for the primary electron transfer to occur, the $L$ and $M$ branches (see figure).

For reasons which are not fully understood, the pathway of choice is $\mathrm{L}$. The primary donor $\mathrm{P}$ is a dimeric form of bacteriochlorophyll $(\mathrm{BChl})$, and is shared by both pathways. In addition to this, there are two monomeric bacteriochlorophylls $\left(B_{L}\right.$ and $B_{M}$ ) and two bacteriopheophytins $\left(\mathrm{H}_{\mathrm{L}}\right.$ and $\left.\mathrm{H}_{M}\right)$. The secondary electron acceptors are bound quinone molecules called $Q_{A}$ and $Q_{B}$. The donor $\left(D_{1}\right)$ to $\mathrm{P}^{+}$is the haem of a cytochrome which may be bound to the reaction centre, as in the case of Rps. viridis, or may be a soluble protein, as in the case of $R b$. sphaeroides.

According to the crystal structure (see figure), it would be logical if $A_{1}$ was $B_{L}$, but the data of Fleming et al. in this issue do not support this idea. Using stimulated emission in the infrared to monitor the $\mathrm{PA}_{1}$ to $\mathrm{P}^{+} \mathrm{A}_{1}^{-}$transition in both $R p s$. viridis and $R b$. sphaeroides, they conclude that $A_{1}$ is the bacteriopheophytin $H_{L}$. According to their data, if there is an involvement of $B_{L}$ in the electron exchange between $\mathrm{P}$ and $\mathrm{H}_{\mathrm{L}}$, then it must include an adiabatic step or be incorporated into a superexchange mechanism with very strong quantum mechanical coupling. Relevant to these possibilities is the finding that rate of primary charge separation increases with decreasing temperatures. At room temperature, the rate for $\mathrm{P}^{+} \mathrm{A}_{\text {- }}^{-}$formation is identical for both bacterial systems, but at $8 \mathrm{~K}$ not only does the rate increase dramatically but it is almost twice as fast in Rps. viridis as it is in $R b$. sphaeroides.

\section{Discrepancy}

Although Fleming et al. ${ }^{1}$ try hard to explain these data in terms of conventional theory for non-adiabatic electron transfer, they are forced to conclude that the validity of the arguments is not convincing, particularly in the case of Rps. viridis. The problem is that the components involved in primary charge separation are embedded in a protein environment ${ }^{3.4}$, and it is this complication which probably underlies the discrepancy between theory and the differences obtained with the two bacterial systems. Indeed, the crystallographic data indicate small differences in organization of the chromophore within the Rps. viridis and $R b$. sphaeroides reaction centres ${ }^{3.4}$, the implications of which are not fully understood in terms of the kinetics of primary charge separation. Also, the static structure obtained by X-ray diffraction does not reveal the conformational changes that may occur when the reaction centre is activated by light.

Clearly, Fleming et al. provide the necessary experimental data which, together with the crystallographic structures, will allow theoreticians to modify existing ideas and to develop new ones about how photosynthetic organisms so efficiently bring about the fastest bimolecular reactions known in photochemistry.

1. Fleming, G. R., Martin, J. L. \& Breton, J. Nature 333, 190192 (1988)

2. Deisenhofer, J., Epp, O., Miki, K., Huber, R. \& Michel, H. J. molec. Biol. 180, 385-398 (1984).

3. Allen, J. P., Feher, G., Yeates, T. O., Komiya, H. \& Rees, D. C. Proc. natn. Acad. Sci. U.S. A. 84, 5730-5734 (1987) 4. Deisenhofer, J., Epp, O., Miki, K., Huber, R. \& Michel, H. Nature 318, 618-624 (1985).

Jim Barber is Professor of Plant Physiology and Head of the AFRC Photosynthesis Research Group, Imperial College of Science and Technology, London SW7 $2 B B, U K$. 